\title{
Spontaneous pneumomediastinum (Hamman's syndrome) following a vaginal delivery
}

\author{
Harjit S Dhaliwal \\ Correspondence: Dr Harjit S Dhaliwal, Department of Obstetrics and Gynaecology, \\ Wrexham Maelor Hospital, Croesnewydd Road, Wrexham LL137TD, Wales; Email - \\ harjitdhaliwal2004@yahoo.co.uk \\ Distributed under Attribution - NonCommercial - Share Alike 4.0 International (CC BY-NC-SA 4.0)
}

\begin{abstract}
A 22 year old primigravida was reviewed on day 1 following a normal vaginal delivery. The patient reported neck swelling and pain. The pain was exacerbated by movement. She was not suffering from any chest pain nor had difficulties with breathing or swallowing. Diagnosis of spontaneous pneumomediastinum was made. Vaginal delivery complicated by pneumomediastinum and subcutaneous emphysema is rare, with a reported incidence as low as 1 in 100,000 deliveries. Treatment of Hamman's syndrome is supportive and the course is self- limiting.
\end{abstract}

Keywords: Postpartum pneumomediastinum, spontaneous pneumomediastinum, mediastinal emphysema, subcutaneous emphysema.

Hamman's syndrome (subcutaneous emphysema and pneumomediastinum) is a rare complication of labour and delivery. Spontaneous pneumomediastinum is usually a benign self limiting illness. It usually occurs in the second stage of labour; nevertheless, it is often clinically apparent only in the postpartum period. ${ }^{1-4}$ It has been reported in all stages of labour and has been related to hyperemesis gravidarum and self-induced vomiting. ${ }^{2}$ It was first described by Louis Hamman in 1939. The reported incidence is 1 in 100,000 deliveries. About 200 cases have been reported in the literature in the last 400 years. ${ }^{5}$ Chest pain is the most common symptom of pneumomediastinum although the severity depends on the location and the amount of air present. Additional symptoms may include dyspnoea, cough and palpitations, chest tightness, neck pain or discomfort, dysphagia and numbness of hands. Subcutaneous emphysema of the neck and face with crepitations are virtually pathognomonic ${ }^{2,6-8}$. Subcutaneous emphysema especially of the neck has a reported prevalence ranging from 40$100 \%$ and the most commonly reported sign ${ }^{9-10}$.

\section{Case report}

On booking at 12 weeks gestation the patient was allocated to midwifery led care. Her expected date of delivery was confirmed by ultrasound. It was noted that the patient had a body mass index of $25.8 \mathrm{~kg} / \mathrm{m}^{2}$. She was an ex-smoker, who gave up smoking more than 12 months prior to conception. Antenatal blood and midstream urine investigations were all normal. The quadruple screening test was done at 16 weeks suggested

Received: $13^{\text {th }}$ May 2019. Accepted: $13^{\text {th }}$ June 2019.

Dhaliwal HS. Spontaneous pneumomediastinum (Hamman's syndrome) following a vaginal delivery. The New Indian Journal of OBGYN. 2019; 6(1): 67-70. 
a low risk $(1: 10,000)$. She had a routine foetal anomaly scan at 19 weeks gestation with no abnormalities detected. Placental site was posterior, not low with a three vessel cord seen. She was classed as low risk and had her entire antenatal care out in the community. The GROW (Gestation Related Optimal Weight) chart which was customised for her in the pregnancy, suggested a linear growth based on fundal height measurements.

She was referred to the day assessment unit at 40 weeks and 4 days with a raised blood pressure and symptoms of labour; however a blood pressure profile was normal, urine dipstick was negative and her uterine activity corresponded with latent phase of labour. The patient was counselled and sent home with analgesia. She was advised to await events.

At 40 weeks and 5 days she presented in established labour. She progressed normally through labour and had an uneventful vaginal delivery. The analgesia used in labour was entonox (nitrous oxide and oxygen). There was an estimated documented blood loss of $140 \mathrm{ml}$. Duration of the first stage of delivery was $7 \mathrm{hrs}$ and 15 mins, second stage was 58 mins and completion of the third stage was 8 mins. A live male baby was delivered with a birth weight of $3680 \mathrm{Kg}$. The neonate had Apgar scores of 8 at $1 \mathrm{~min}$ and 9 at 5 mins. Meconium was present at delivery and baby responded well to warming .The neonate was to have 12 hours of meconium observations as per unit policy. It was noted that the patient had low grade pyrexia during the second stage of labour. No action was taken and her temperature reverted back to normal post delivery. The immediate postpartum period was unremarkable.

About 8 hours after delivery the patient complained of a swollen throat, with soreness and tightness of the neck. It was worse on turning her head. No breathing difficulty was reported. She reported a sensation of a 'pulled muscle' mainly on the right side which started whilst pushing in labour. On examination of the throat; the uvula was central, the tonsils normal and there were no signs of infection. On palpation for cervical lymphadenopathy there was marked 'crunching' felt beneath the skin of the neck; similar to surgical emphysema. For completeness, the chest was examined, which showed good air entry and was clear bilaterally. The heart sounds were normal. Baseline blood investigations were performed. The bloods showed a raised white blood cell count 18.9 thousand per cumm consisting mainly of neutrophils 15.6 thousand per cumm and monocytes 1.6 thousand per cumm. The $\mathrm{C}$ reactive protein $(\mathrm{CRP})$ of $139(<5)$ was also significantly raised. On day 2, her range of neck movements were marginally better. Her C - reactive protein dropped to $113(<5)$ and a chest X-ray $(\mathrm{CXR})$ was performed (Figure 1). In $X$ ray report, there was significant surgical emphysema in the right soft tissues over the

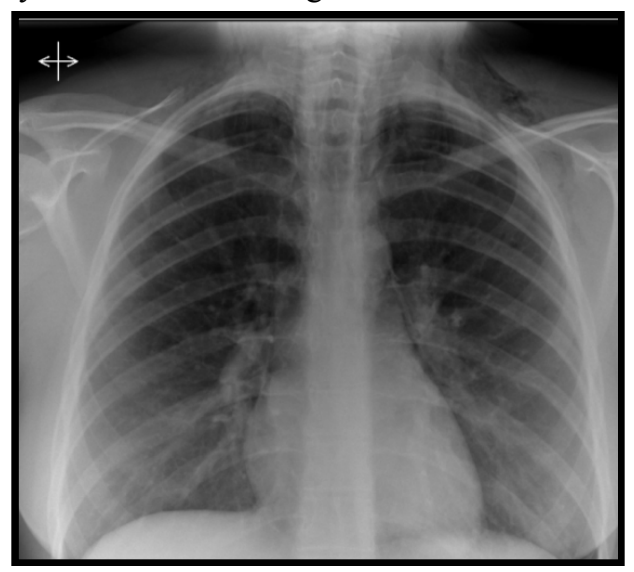

Figure 1: Day 2 chest $X$ ray

supraclavicular fossae bilaterally. There was a pneumomediastinum. No obvious pneumothorax was identified. No pleural effusion was found. Lungs were clear. The patient was reviewed by our medical colleagues who agreed with an initially diagnosis of Hamman's Syndrome. On Day 3, a repeat chest X ray was

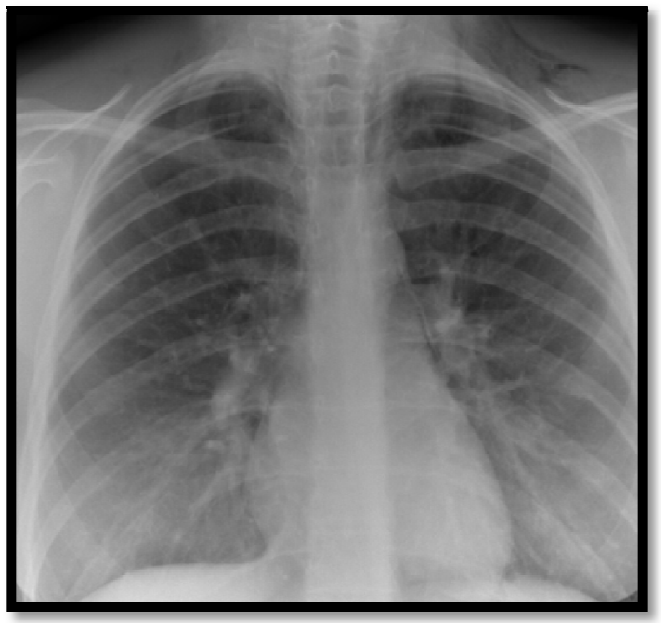

Figure 2: Chest $X$ ray day 3 
performed. This radiograph was reported as the subcutaneous emphysema appears improved with no evidence for any pneumothorax or other interval abnormality (Figure 2). Our cardiology colleagues reviewed the patient and then performed a bedside echocardiogram and it was reported as normal. Initial management consisted of regular nonsteroidal antiinflammatory analgesia. Following the chest $\mathrm{X}$ ray and review by the medical team; the patient was put on intravenous antibiotics as a differential diagnosis of mediastinitis was also considered. The WBC and CRP gradual fell to within normal limits following 3 days of treatment and were switched to a course of oral antibiotics on discharge.

\section{Discussion}

The occurrence of Hamman's syndrome in obstetrics is related to the valsalva (forced expiration against a closed glottis) manoeuvre during the expulsive phase of labour. The "pushing down" phase is related to an increase in intra-alveolar pressure which may in turn lead to alveolar rupture. The barotrauma causes alveolar rupture, which then allows air to flow down a pressure gradient from the alveolus to the mediastinum via pulmonary interstitium and pulmonary hilum. Alveolar rupture leads to the accumulation of air in the interstitium and mediastinum (Macklin effect). Once in the mediastinum, the air decompresses in to the cervical space, soft tissues, or even the retroperitoneal space. This third mechanism is the pathophysiological basis behind that of spontaneous pneumomediastinum. Other reported mechanisms include oesophageal rupture during childbirth, or dissection of a pneumoperitoneum ${ }^{5}$, secondary to an epidural catheter placement or caesarean section. Pneumothorax may coexist when air passes between visceral and parietal pleura. ${ }^{6}$ Precipitating causes of the valsalva manoeuvre described to cause spontaneous pneumomediastium are coughing, sneezing, vomiting, defecation and blowing up party balloons ${ }^{9,10}$. Alveolar capillary membrane abnormalities can also bring about the development of an alveolar rupture. Reported risk factors, which can predispose to Hamman's syndrome, are; pulmonary emphysema, interstitial lung disease, bronchiectasis, asthma (8-39\%) and lung transplant recipients.
Diagnosis can be rapidly confirmed by chest X-ray with both posterior anterior and lateral views. About 50\% of cases may remain undiagnosed if only a posterior anterior view is taken; hence a lateral view is necessary. About $30 \%$ of patients with spontaneous pneumomediastinum can have a normal chest $\mathrm{x}$-ray. The chest CT scan is considered the gold standard for detecting the mediastinal air, as it can detect small amounts that cannot be seen on chest x-ray. Contrast imaging is useful if the is suspicion of an oesophageal tear (Borerhaave's syndrome). Bronchoscopy is the definitive test when a tracheobronchial tree rupture is suspected. There is also Hamman's sign, which is a crunching, rasping sound, synchronous with heart sounds, auscultated over the precordium. The reported prevalence of Hamman's sign is highly variable. Subcutaneous emphysema in the neck can lead to compression of the trachea and upper respiratory tract obstruction. ECG changes include nonspecific ST and T wave abnormalities have been reported in about $25 \%$ of patients. A serious differential diagnosis should carefully be considered Boerhaave's syndrome. It is a life-threatening condition though relatively rare. Both these conditions are commonly precipitated by the same factors, (coughing, straining and vomiting). They both present with chest pain, dyspnoea, neck pain, pneumomediastinum and surgical emphysema. In Boerhaave's syndrome the patient is unwell with tachycardia, tachypnoea, fever, hypotension and hydropneumothorax. Other potential more life-threatening conditions include; acute coronary syndrome, pericarditis, pneumothorax, aortic dissection and pulmonary embolism and especially tracheobronchial tree ruptue. ${ }^{10}$

The treatment of Hamman's syndrome is mainly supportive. Chest pain, dyspnoea and anxiety can be treated with oxygen, analgesics and sedative therapy. It is usually benign and has a self-limiting course ${ }^{1}$. Reoccurrence is uncommon and rare $^{7}$. Management in subsequent pregnancies is controversial. Some advocate routinely using low forceps, in order to withhold active pushing during the second stage of labour. The use of an epidural has been suggested as it may effectively control the involuntary and active pushing. Entonox (nitrous oxide and oxygen) in labour should be avoided, as its 
The New Indian Journal of OBGYN. 2019 (July-December); 6(1)

properties can lead to substantial expansion of the trapped gases.

\section{Conclusion}

Spontaneous pneumomediastinum and subcutaneous emphysema (Hamman's syndrome) is a rare condition with its potential complications during labour and delivery. Making an accurate early diagnosis is vitally important as its presentation is similar to Boerrhaave's syndrome. Appropriate management in subsequent pregnancies is unclear. Epidural analgesia has been suggested, in order to minimize both involuntary and active maternal pushing.

\section{Conflict of interest: None. Disclaimer: Nil.}

\section{References}

1.Seidl JJ, Brotzman GL. Pneumomediastinum and subcutaneous emphysema following vaginal delivery: case report and review of the literature. Journal of Family Practice. 1994; 39(2): 178-80.

2.Karson EM, Saltzman D, Davis MR. Pneumomediastinum, and pneumothorax in labour and delivery. Am J Obstst Gynecol. 1986; 154: 487-9.

3.Duffy B. Post partum pneumomediastinum. Anesth Intensive Care. 2004; 32:117-9.
4.Krause H, Portmann C, Pneumomediastiun following vaginal delivery. Aust NZ J Obstet Gynaecol. 2000; 40(1):106-7.

5.Crean PA, Strong JM, FitzGerald MX. Spontaneous Pneumomediastium - Case report. Br J Obstet Gynaecology. 1981; 88(9); 952-4.

6.Bonin MM. Hamman's syndrome (spontaneous pneumomediastinum) in a parturient: a case report. Journal of Obstetrics and Gynaecology Canada. 2016; 28(2):128-31.

7.Tytherleigh MG, Connolly AA, Handa JL. Spontaneous pneumomediastinum. J Accid Emerg Med. 1997; 14(5): $333-4$.

8.Mahboob A, Eckford SD. Hamman's syndrome: An atypical cause of postpartum chest pain. J Obstet Gynaecol. 2008; 28; 652-3.

9.McMahon DJ. Spontaneous pneumomediastinum. Am J Surg. 1976; 131: 550-1.

10.Koulias GJ, Karkolis DP, Wang XJ, Hammond GL. Current assessment and management of spontaneous pneumomedistinum: experience in 24 adult patients. Eur $\mathrm{J}$ Cardiothoracic Surg. 2004; 25: 852-5.
Harjit S Dhaliwal ${ }^{1}$
${ }^{1}$ Senior Registrar/Staff specialist/Department of Obstetrics and Gynaecology, Wrexham Maelor Hospital, Croesnewydd Road, Wrexham LL137TD, Wales. 